\title{
LA GOBERNABILIDAD O CAPACIDAD PARA GOBERNAR. EL PAPEL DEL LIDERAZGO POLÍTICO ${ }^{1}$
}

\author{
Maria Fatima Pinho De Oliveira ${ }^{2}$
}

\section{Resumen}

El presente trabajo tiene como objetivo analizar el concepto de gobernabilidad, visto desde la perspectiva de teóricos como Alcántara, Arbós y Giner, Huntington, Bell, Nohlen y Neudstadt, entre otros. La gobernabilidad se ha definido como la capacidad de gobernar, y esta capacidad puede ser limitada por las crisis que de los sistemas democráticos que vienen dadas por factores tanto internos (partidos políticos, sociedad) como factores externos (entorno mundial), donde la eficacia y la legitimidad juegan un papel importante dentro del sistema. En cuanto a la forma de evaluación del líder a través del liderazgo, es muy importante el papel que juega el Presidente como líder, ya que a medida que surjan las crisis se medirá la capacidad de su liderazgo así como el rol de los actores, las medidas aplicadas y el énfasis en sus seguidores.

Palabras Claves: Gobernabilidad; Líder; Gerencia; Democracia Y Ciencia Política

\section{INTRODUCCIÓN}

El término gobernabilidad tiene varias acepciones, en materia marítima se refiere a la capacidad del capitán y su tripulación que van conduciendo una nave y sus pasajeros a un puerto de destino; también es referido a la conducción del Estado. La gobernabilidad entonces puede entenderse como la capacidad y calidad del desempeño del gobierno, así como la necesidad que tienen los gobiernos de lograr una combinación de eficiencia administrativa y económica y de elementos democráticos que permitan ofrecer estabilidad y legitimidad.

El presente trabajo aborda la situación de la gobernabilidad o capacidad de gobernar como una definición establecida desde los años 70 producto de la crisis de sistemas democráticos en la búsqueda del modelo de Estado de bienestar. El problema de la gobernabilidad para los autores Alcántara, Arbós y Giner, Samuel Huntington, Crozier, Watanuki y Daniel Bell, se ha suscitado por la presencia de cambios, resultado de la adopción de políticas neoliberales que redujeron en gran medida el rol que tradicionalmente tenía el Estado.

Los autores antes señalados en el párrafo anterior han mantenido una teoría donde el autor NOHLEN

\footnotetext{
${ }^{1}$ Este manuscrito está elaborado basado en las investigaciones realizadas por la autora cursante del Doctorado en Ciencia Política de la Universidad Simón Bolívar, bajo la revisión y asesoría del profesor PhD. Herbert Koeneke R.

${ }^{2}$ Profesora adscrita al Departamento de Teconologia de Servicios. Doctorando en Ciencia Politica Universidad Simón Bolívar. Email: mpinho@usb.ve
} 
(1994) explica, que la falta de eficacia demostrada por los gobiernos, ha generado una suerte de deslegitimación de los mismos y con ello una pérdida de la confianza en el sistema democrático; es por ello que se hace necesario solucionar el dilema del rol que le compete jugar al Estado, a partir de las relaciones que puedan establecerse entre los diferentes actores estratégicos para darle continuidad al régimen democrático.

En virtud de lo anterior, el presente trabajo aborda una revisión bibliográfica de los autores que tratan las diversas concepciones teóricas (conservadora, neoconservadora, liberal y marxista) de la gobernabilidad, la legitimidad y eficacia como factores de gobernabilidad, diversas definiciones de la Gobernabilidad, el Problema de la Gobernabilidad y un breve resumen de Neudstadt sobre ¿Líder o Empleado?, es decir, la influencia del Poder Presidencial como servidor del estado.

\section{CONCEPCIONES TEÓRICAS SOBRE LA GOBERNABILIDAD}

Para los autores Xavier ARBÓS y Salvador GINER (1996: 9), citado por HEREDIA (2002: 2), la gobernabilidad puede definirse desde varias perspectivas, dependiendo del punto de vista ideológico de cada autor. Por ello concluyen que existen, al menos, cuatro tipos de orientaciones en el momento de definir el concepto de gobernabilidad: conservadora, neoconservadora, liberal y marxista.

Manuel ALCÁNTARA (2004: 32) coincide con la división realizada por ARBÓS y GINER, aunque para él, las principales corrientes teóricas estarín representadas por los neoconservadores, los liberales y los neomarxistas, la diferencia entre ambas visiones está dada por el factor ideológico que caracteriza la posición de ARBÓS y GINER, puesto que son ellos mismos quienes aclaran que:

Si simplificamos, podemos presentar a los conservadores como los partidarios de un equilibrio entre Estado y mercado que sea favorable al mercado, los neoconservadores son por principio contrarios a cualquier acción estatal que vaya más allá de la garantía de los derechos individuales: cualquier intervencionismo en la economía es sospechoso de llevar el germen del totalitarismo.

En cambio para los conservadores como Samuel Huntington, Crozier, Watanuki y Daniel Bell, entre otros, citados por HEREDIA (2002: 2), la crisis de gobernabilidad por la que atravesaron los países desarrollados a finales de los años '60 y comienzo de los ‘70, fue el resultado lógico de la excesiva intervención estatal en la economía de mercado. Esto se explica por la aparición de una serie de movimientos que solicitaban una mayor participación en la actividad democrática, obligando a los gobiernos a responder con medidas de carácter social a objeto de poder contener las presiones ejercidas por estas organizaciones. Esta corriente referida a la gobernabilidad explican su posición negativa (la ingobernabilidad) como el producto de una sobrecarga de demandas a las que el Estado respondía con expansión de sus servicios y de su intervención provocando inevitablemente una crisis fiscal. (ALCÁNTARA, 2004: 33). Para los conservadores la gobernabilidad sólo se logra en la medida en que se evitan todas aquellas actividades de gobierno que son vistas como atentatorias contra 
el mercado.

Los autores conservadores como Nozick o Hayek que se diferencian de los primeros por ser más ideológicos, ven la crisis de gobernabilidad como un problema relativo a las interferencias que recibe la sociedad del Estado, desde el exterior (ARBÓS y GINER, 1996: 9).

Criticando esta ideología socialista, Friedrich A. HAYEK (1995: 95), citado por HEREDIA (2002) plantea que:

... sólo podemos contar con un acuerdo voluntario para guiar la acción del Estado cuando ésta se limita a las esferas en que el acuerdo existe... Cuando el sector comunal, en el que el Estado domina todos los medios, llega a sobrepasar una cierta proporción de la totalidad, los efectos de sus acciones dominan el sistema entero ....

Para los conservadores, el Estado no debe tener ninguna otra actividad que vaya más allá de la garantía de los derechos individuales, por lo que el Estado no está llamado a intervenir en asuntos de carácter económico, por ejemplo la libre competencia, en este sentido HAYEK (1995: 66-68), reconoce que:

... tampoco son incompatibles el mantenimiento de la competencia y un extenso sistema de servicios sociales, en tanto que la organización de estos servicios no se dirija a hacer inefectiva en campos extensos de la competencia... [ya que] ...en ningún sistema que pueda ser defendido racionalmente el Estado carecerá de todo quehacer. Un eficaz sistema de competencia necesita, tanto como cualquier otro, una estructura legal inteligentemente trazada y ajustada continuamente....

HAYEK (1995: 101), señala que ni el colectivismo ni el aumento de las prestaciones sociales por parte del Estado, son argumentos válidos que pudieran ser utilizados para socavar el basamento teórico de la democracia capitalista contemporánea cuando señala:

... se dice ahora con frecuencia que la democracia no tolerará el «capitalismo». Por ello se hace todavía más importante comprender que sólo dentro de este sistema es posible la democracia, si por «capitalismo» se entiende un sistema de competencia basado sobre la libre disposición de la propiedad privada. Cuando llegue a ser dominada por un credo colectivista, la democracia se destruirá a sí misma inevitablemente.

En cuanto a los liberales, el problema se centra en cómo restaurar la autonomía política de los Estados sin que ello afecte al mercado ya que éste debe ser necesariamente libre. Los representantes de ésta teoría -Buchanan, Wagner, Brittan- sostienen que la crisis de gobernabilidad respondió a la mala relación existente entre la política económica keynesiana y la democracia. BUCHANAN y WAGNER (1983: 126) indican que se trata de la “...yuxtaposición de las prescripciones de la política keynesiana y la democracia política crean una mezcla inestable. El orden económico parece hacerse cada vez más frágil, llegando a parecer un castillo de naipes ...”. Por ello en la medida en que el Estado interviene en la economía se pierde la independencia que debe existir entre la libertad política y la libertad económica, generándose los problemas de ingobernabilidad derivados de la alta inflación generadas por las presiones que sufre la democracia, que ven al Estado como un prestador de servicios inagotables.

Para ARBÓS y GINER (1996: 11) citado por HEREDIA (2002), “... el problema de la gobernabilidad, 
desde la perspectiva liberal, será el de restaurar la autonomía de la política restringiendo su alcance hasta el límite del mercado para, simultáneamente, reconstruir la libertad de éste ...”.

Los marxistas, consideran que el problema de la gobernabilidad está centrado en la propiedad de los medios de producción, porque los gobiernos siempre defenderán los intereses del capital, sin importar que esta defensa traiga aparejada la inevitable crisis fiscal (ARBÓS y GINER, 1996: 9-13). El autor HUNTINGTON (1975: 73) reconoce que "... el aumento del déficit fiscal, y sus efectos evidentemente desestabilizadores fueron suficientemente desastrosos para la existencia del sistema como para generar una nueva variedad en el análisis marxista sobre la inevitabilidad del colapso capitalista ...".

El autor O'CONNOR con su teoría, reforzó esta perspectiva al señalar que la crisis fiscal del Estado capitalista no era otra cosa que la inevitable consecuencia debida a la brecha estructural entre los gastos estatales y las rentas (HEREDIA, 2002: 4).

Para O'CONNOR (1981: 205), la crisis de legitimación por la que atravesaba el Estado en la década de los ' 60 y '70, unida al deterioro del nivel de vida de los trabajadores del sector competitivo, produjo el despertar de la población excedente obligándola a adoptar un fuerte activismo político que intentó ser detenido por la proliferación de una gran variedad de programas estatales que se enmarcaban dentro de las actividades del Estado benefactor.

No obstante, para O'Connor, 1981, esta interferencia no fue de gran ayuda lo que lo lleva a proclamar el fracaso del Estado benefactor, minando con ello la única fuente de legitimación del Estado contemporáneo; si el aparato estatal no puede ofrecer bienes y servicios que se consideran indispensables desde hace medio siglo, cae por la base su pretensión de legitimidad, donde las causas de la ingobernabilidad responden a la incapacidad fiscal del Estado para absorber las necesidades de una sociedad democrática (HEREDIA, 2002: 4).

Para concluir en palabras de HEREDIA (2002), la gobernabilidad se relaciona con dos factores que la sustentan, transformándose en las variables independientes que se deben considerar en todo estudio relacionado con la gobernabilidad. Estos factores son la legitimidad y la eficacia.

\section{LEGITIMIDAD Y EFICACIA COMO FACTORES DE GOBERNABILIDAD}

Muchos autores presentan definiciones, una de las más resaltantes es la de Max WEBER, que sostiene que el poder debe estar basado en la legitimidad, WEBER (1994: 174), en cuanto al tema de la dominación resalta que el poder está basado en la legitimidad, ésta nace de la razón y descansa en la creencia de la legalidad de las acciones estatuidas y de los derechos del mando de los llamados a ejercer la autoridad.

Para el autor MORLINO, (1985), la legitimidad “... consiste en un conjunto de actitudes positivas hacia el sistema político considerado como merecedor de apoyo..." (MORLINO, 1985: 177), apreciación que 
también es compartida por otros autores cuando sostienen que la legitimidad está dada por " ... la capacidad de un sistema político para generar y mantener la convicción de que las instituciones políticas existentes son las más convenientes o apropiadas para la sociedad..." (LIPSET, 1992: 130), o por la confianza o el apoyo que la población tenga respecto de un determinado régimen (ALCÁNTARA, 2004). Entonces en un régimen democrático la legitimidad puede ser entendida como "... un conjunto de actitudes positivas de una sociedad hacia sus instituciones democráticas, consideradas como la forma de gobierno más apropiada ..." (MONTERO y MORLINO, 1993: 9).

Juan LINZ (1996) citado por HEREDIA (2002) señala que este tipo de régimen debe buscar la obediencia, pero no una obediencia en base a la costumbre. Weber argumenta que la costumbre y la situación de intereses, no menos que los motivos puramente efectivos y de valor (racionales con arreglo a valores) no pueden representar los fundamentos en que la dominación confía. Y a esto se les añade otro factor: la creencia en la legitimidad, esa creencia es la que permite que un régimen democrático pueda exigir obediencia para hacer cumplir una decisión y debe ser debe ser compartida por una mayoría de los ciudadanos sobre todo por aquellos que controlan a las fuerzas armadas.

Es por lo anterior que: "... la legitimidad de un régimen democrático se apoya en la creencia en el derecho de los que han llegado legalmente a la autoridad para dar cierto tipo de órdenes, esperar obediencia y hacerlas cumplir, si es necesario, utilizando la fuerza ..." (LINZ, 1996: 38). Esto lo pueden hacer debido a que han llegado al poder a través de elecciones libres, mecanismo por medio del cual, el resto de la sociedad les ha otorgado un reconocido y sistemático derecho a gobernar (LIPSET, 1996: 23).

Según Victoria CAMPS (1997: 23) citada por HEREDIA (2002), señala que la legitimidad es algo muy difícil de probar empíricamente, y que para una democracia para no pierda la legitimidad, es necesario que los gobiernos mantengan ciertas reglas, tales como:

1. Fidelidad y respeto a la constitución;

2. Fidelidad al propio programa político;

3. Ejecución del programa a través de medios que permitan alcanzar los objetivos propuestos.

Por lo tanto, "... el no cumplimiento de lo previsto, la incoherencia entre lo que se dijo que se haría y lo que se hace de hecho, o entre lo que ideológicamente se espera de un partido y lo que ese mismo partido demuestra que cree, es lo que determina la pérdida de legitimidad sustantiva de un gobierno..." (CAMPS, 1997: 23).

Se puede decir entonces que la legitimidad es una cualidad de la gobernabilidad; la estabilidad tiene que ver con el estado de la gobernabilidad y la eficacia/eficiencia es una propiedad de la gobernabilidad. Así, cuando se aborda la relación entre gobernabilidad y eficacia, la gobernabilidad es pensada como una propiedad de los 
sistemas políticos definida por su capacidad para alcanzar objetivos prefijados al menor costo posible. Cuando se trata de la relación entre gobernabilidad y legitimidad la atención se refiere al problema de la calidad de la acción gubernamental. Y cuando la relación se constituye entre estabilidad y gobernabilidad se apunta a la previsible capacidad del sistema de durar en el tiempo puesto que un sistema es estable cuando tiene capacidad para transformarse a través de su adaptación a los desafíos que provienen de su entorno. (CAMOU, 2001: 33-35)

Para MORLINO, citado por HEREDIA (2002), la eficacia -que él la denomina como eficacia decisoriaes:

...la capacidad que tiene un régimen o, mejor, que tienen las estructuras del mismo para tomar y ejecutar las decisiones necesarias para superar los retos planteados al régimen o las otras medidas destinadas a alcanzar los diversos fines requeridos por los gobernantes, el primero de los cuales es, de ordinario, el mantenimiento del propio régimen (MORLINO, 1985: 219).

LINZ (1996: 46), define la eficacia como:

... la capacidad de un régimen para encontrar soluciones a los problemas básicos con los que se enfrenta todo sistema político (y los que cobran importancia en un momento histórico), que son percibidos más como satisfactorias que como insatisfactorias por los ciudadanos conscientes.

Seymour Martin LIPSET (1992: 115) en consonancia con los autores anteriores, señala que:

...por eficacia se entiende la actuación concreta de un sistema político: en qué medida cumple las funciones básicas de gobierno, tal y como las definen las expectativas de la mayoría de los miembros de una sociedad y las de los grupos poderosos que hay dentro de ella, que podrían constituir una amenaza para el sistema como, por ejemplo, las fuerzas armadas. La eficacia de un sistema político democrático, caracterizado por una burocracia eficiente y un sistema de decisiones capaz de resolver problemas políticos, puede diferenciarse de la eficacia del sistema considerado en su totalidad, aunque, por supuesto, el fracaso del funcionamiento de la sociedad en su conjunto influirá en el subsistema político.

Manuel ALCÁNTARA (1994: 18) señala que la eficacia apunta al grado de cumplimiento de las expectativas y las necesidades de la población, lo que se expresa a través de dos aspectos:

... en cuanto a la idoneidad de las políticas diseñadas por el gobierno para reducir al mínimo el conflicto, asegurar una tendencia a la repartición de la riqueza y propiciar su incremento; y con respecto a la capacidad operativa del gobierno de actuar sin sobresaltos, pudiendo realizar sus programas y no estando sujeto a elementos disfuncionales.

En resumen, la eficacia es la capacidad que tienen los gobiernos para satisfacer las demandas básicas de la población, directamente relacionadas con el "... desempeño real del gobierno y de los grupos de poder...” (LIPSET, 1992: 41).

De lo anterior se puede deducir que no hay diferencias entre las definiciones de legitimidad y eficacia ya que sus características resaltantes refieren primeramente a un conjunto de valores basados en la creencia en que un sistema debe ser de una determinada manera porque no existe otro mejor, y en segundo lugar a las exigencias que los miembros de ese sistema hacen a las autoridades que los rigen; según el autor HEREDIA (2002), aquí se pueden buscar las respuestas a los problemas de la gobernabilidad que presentan los países desarrollados en la década de los '70 que también afectaron las naciones latinoamericanas quienes iniciaron sus procesos de 
democratización en la década de los ' 80 .

Para finalizar, los autores ARBÓS y SARASA (1997: 9) señalan que:

\begin{abstract}
... la legitimidad de nuestras autoridades, hoy, no depende tan sólo de las urnas, sino de su capacidad de rendir ciertos servicios públicos, así como la de servir de uno u otro modo los intereses de la ciudadanía en general y, en especial, la de aquellos sectores de la población más necesitados de asistencia. Sin educación universal pública, tributos progresivos, subsidios de desempleo y jubilación, más varias otras prestaciones o medidas de aplicación general -y sin duda, imperfecta- no se justifica ya un gobierno moderno que se pretenda civilizado.
\end{abstract}

\title{
LA GOBERNABILIDAD
}

En el caso de la gobernabilidad se cita al autor Manuel ALCÁNTARA (1994: 11), que entiende “...p por gobernabilidad la situación en que concurren un conjunto de condiciones favorables para la acción de gobierno que se sitúan en su entorno (de carácter medioambiental) o que son intrínsecos a éste ..." . La misma se forma a partir de impulsos que vienen del propio gobierno y que se expresan a través de las políticas públicas, éstas a su vez son tomadas por la sociedad, que emite su respuesta a través de individuos solos o a través de las organizaciones (ALCÁNTARA, 1994: 22). El objetivo supone mantener el buen rendimiento de las instituciones con el fin de asegurar la gobernabilidad del sistema.

NOHLEN, citado por HEREDIA (2002: 4), explicó algo similar a lo dicho por ALCÁNTARA indicando que:

... el concepto de gobernabilidad se refiere a la interacción entre gobernantes y gobernados, entre capacidades de gobierno y demandas políticas de gobierno. Hace referencia a la tensión que existe entre las dos partes y pone en cuestión el sistema de gobierno, como productor de decisiones políticas y encargado de su ejecución, y su capacidad para estar a la altura de los problemas a resolver.

El autor Rolando FRANCO (1994: 48) explica que la gobernabilidad es un concepto en el cual se encuentra inmersa la voluntad política de querer hacer algo, aunque esta voluntad no lo es todo, ya que hay que saber cómo hacer las cosas (ese algo), el autor expresa que:

Esto último tiene que ver con la capacidad de gobernar, que nos es más que, poseer la condición para diseñar y ejecutar políticas públicas y lograr efectividad en el desenvolvimiento de la actividad gubernamental. Los componentes cruciales de dicha capacidad son un poder gubernamental adecuado (lo que implica reformas institucionales) y la existencia de consenso social (legitimación del régimen político y del gobierno), además de elementos operativos que se logran mediante el perfeccionamiento de las élites políticas. Es necesario también que el público se identifique con las estrategias de desarrollo, lo que exige ilustrar a la población, entregándole visiones realistas del proceso. (FRANCO, 1994: 48)

De las tres (3) definiciones anteriores, los autores se basan en el accionar gubernamental. Aunque para otros autores la acción de los gobiernos debe ir acompañada de la aceptación social, por ello es importante la legitimidad, por ejemplo: 
... la gobernabilidad es la cualidad propia de una comunidad política según la cual sus instituciones de gobierno actúan eficazmente dentro de su espacio de un modo considerado legítimo por la ciudadanía, permitiendo así el libre ejercicio de la voluntad política del poder ejecutivo mediante la obediencia cívica del pueblo. ARBÓS y GINER (1996: 13)

Por su parte, Ángel FLISFISCH citado por HEREDIA (2002) define a la gobernabilidad como:

... la calidad del desempeño gubernamental a través del tiempo, situación que se logra a través de la capacidad de adoptar oportunamente decisiones ante eventos que son desafíos que exigen una respuesta gubernamental; efectividad y eficiencia de las decisiones adoptadas; aceptación social de esas decisiones; y coherencia de las decisiones a través del tiempo, de manera que no produzcan efectos contradictorios.

Luciano TOMASSINI (1996: 50), agrega que la gobernabilidad “... no sólo se refiere al ejercicio del gobierno, sino a todas las condiciones necesarias para que ésta función pueda desempeñarse con eficacia, legitimidad y respaldo social...”. Destaca que la gobernabilidad consiste en la capacidad del gobierno y de la sociedad para atender equilibradamente, en grados y durante períodos razonables, la triple demanda ciudadana por crecimiento, equidad y democracia, con transparencia a partir de los consensos necesarios y con la más alta participación posible.

Para HEREDIA (2002), ello supone una gran posibilidad para formular políticas que aquellos consensos reflejen: prácticamente todos los estudios publicados en los últimos años en torno a estos problemas coinciden en privilegiar estos elementos así como la concatenación existente entre ellos.

Para los autores ARBÓS y GINER, constatan que existen mínimo cuatro (4) niveles donde se mueven los procesos de gobernabilidad. Se trata del (1) dilema legitimidad-eficacia; (2) de las presiones y demandas del entorno gubernamental, o de la carga de responsabilidades; (3) de la restructuración corporativa de la sociedad civil, y (4) de la expansión y cambio tecnológico, con sus repercusiones demográficas, ecológicas y sociales consiguientes.

ALCÁNTARA (1994: 31) concluye que la gobernabilidad depende de tres (3) variables principales: primero, el fortalecimiento de la sociedad civil y la evolución de la cultura política; segundo, la orientación y el comportamiento de la economía, y tercero, la integración de sectores cada vez más amplios de la sociedad en el sistema productivo.

Para el autor Edelberto TORRES SILVA (1994: 29-30) citado por HEREDIA (2002), señala que la gobernabilidad:

...es la cualidad del sistema político según la cual sus instituciones actúan eficientemente, de un modo considerado legítimo por la ciudadanía, porque les proporcionan seguridad, integración y prosperidad, permitiendo el libre ejercicio de la voluntad política democrática del Estado, así como la participación de los ciudadanos, todo lo cual asegura el orden y la continuidad del sistema.

Por su parte el autor Fernando ZUMBADO (1994: 20) sostiene que el buen funcionamiento de un gobierno depende de la gobernabilidad de la sociedad, concibiendo por esta última como “... las relaciones del Estado, por una parte, y la cultura cívica, la economía y los sectores sociales, por otra ...”. 
En resumen puede considerarse sobre los conceptos de los autores que la eficacia es un factor de gobernabilidad, donde los gobiernos deben orientar su ejecución dentro de parámetros considerado legítimo por la ciudadanía, pues sólo de esta forma podrán alcanzar la aceptación social.

\section{EL PROBLEMA DE LA GOBERNABILIDAD}

El problema de la gobernabilidad, para Gianfranco PASQUINO (1997: 35) está relacionado con dos (2) elementos:

1. El primero de ellos y el más importante es el de que se debe considerar la formulación de políticas eficazy sujeta a la rendición de cuentas una parte integrante de toda solución al problema de la gobernabilidad;

2. El segundo, es que los partidos son los organismos principales en el proceso de formulación de políticas y no pueden ser sustituidos fácil ni eficazmente por otras estructuras. Por consiguiente, siempre que los partidos no puedan, por la razón que sea, aportar ese tipo de formulación de políticas, la crisis de la gobernabilidad volverá a materializarse en formas diferentes, aunque previsibles.

Para el autor citado, aunque se clasifique a la crisis de la gobernabilidad como crisis del capitalismo, como crisis socioeconómica o como crisis de la democracia, es evidente que muy poco se aborda de la labor de los partidos políticos, como agentes esenciales para el funcionamiento de los sistemas políticos. Indica el autor que “... la relación entre gobernabilidad y formulación de políticas no se puede iluminar sin tener en cuenta en serio la vinculación aportada por los partidos políticos ...” (PASQUINO, 1997: 39).

Como a él le interesa el rol de los partidos políticos como factor de gobernabilidad, argumenta que “... la amenaza a la gobernabilidad del sistema y al papel de los partidos puede proceder de dentro o de fuera del sistema político..." (PASQUINO, 1997: 43).

La autora Victoria CAMPS (1997), describe a la gobernabilidad como:

... el proceso constante de hacer frente a las tensiones entre las necesidades crecientes (situaciones problemáticas o aprovechamiento de oportunidades), de una parte, y las capacidades de gobernar (crear tipos de solución o desarrollar estrategias), de otra. Es decir, gobernar no consiste sólo en realizar los llamados actos de gobierno, sino en la interacción palabra clave- entre actores sociales, grupos y fuerzas y las organizaciones e instituciones públicas o semipúblicas. (CAMPS, 1997: 19).

Edgardo BOENINGER (1997: 13) señala que la gobernabilidad es “... la capacidad de una sociedad de gobernarse a sí misma...”, depende de la “... existencia de condiciones para mantener en un país un nivel satisfactorio de estabilidad política, progreso económico y paz social ...”.

En el mismo orden de ideas, explica que existen cinco (5) condiciones:

1. La primera condición para la gobernabilidad es reconocer que dada la situación actual del mundo, existen ciertas limitaciones que condicionan la soberanía y el poder (que se expresa por el menor grado 
de libertad para adoptar las decisiones de política interna), y que por lo tanto, se deben adecuar las instituciones políticas y sociales a esta realidad.

2. La segunda, es la aceptación de la gradualidad del cambio, a objeto de que se produzca una continuidad política, económica y social.

3. La tercera condición está dada por el hecho de que debe existir una conciencia social por el desarrollo y la democracia, es decir, que exista una mayoría que permita llevar adelante un desarrollo sostenido dentro de la estabilidad que otorga un sistema democrático.

4. La cuarta condición, debe existir una mayoría política capaz de gobernar realmente, tomar decisiones, llevarlas a cabo y hacerlas efectivas.

5. Y la quinta condición es concretar la compatibilidad entre crecimiento y equidad.

Para la gobernabilidad en democracia se hace imprescindible la existencia de partidos políticos sólidos y con raíces reales en la sociedad, para que se dé la gobernabilidad es preciso:

1. Fortalecer la organización y la participación ciudadana, para facilitar la existencia de una coalición social y de una coalición política de gobierno;

2. Reforzar la capacidad del ejercicio legítimo de la autoridad;

3. Reforzar la capacidad para prevenir y resolver conflictos y para prevenir y resolver los problemas que afectan a los derechos de las personas. (BOENINGER, 1994: 33).

De los tres autores citados, Pasquino, Camps y Boeninger se puede inferir que tratan la gobernabilidad como un concepto en el que están inmersos actores sociales y públicos, es decir que se refiere a la interacción que se da entre estos actores en función del rol que cada uno de ellos debe jugar en un sistema democrático.

Según HEREDIA (2002), si la gobernabilidad es el resultado de la interacción entre estos actores y de la posibilidad que éstos tienen para ir adecuándose a los cambios que se van produciendo en el conjunto de la sociedad, se abre paso entonces la definición de Michel COPPEDGE quien es -a juicio de Heredia- el que coloca mucho más énfasis en estas relaciones.

El autor COPPEDGE (1994), señala en su definición que incluye directamente a la sociedad civil, es decir, la gobernabilidad puede ser entendida sólo si se analizan las relaciones que se dan entre los actores estratégicos, (las Fuerzas Armadas, los gremios, los sindicatos, la Iglesia, los medios de comunicación) y el propio gobierno; así la gobernabilidad puede definirse como el grado en el cual el sistema político se institucionaliza.

Para HUNTINGTON, (1968) la institucionalización es el proceso por el cual las organizaciones y los procedimientos adquieren valor y estabilidad, se refiere a la frecuencia y al grado en que las organizaciones y procedimientos cambian, la gobernabilidad debe definirse en términos de poder y destaca los siguientes recursos: 
El concepto planteado aquí es una síntesis de diversas aproximaciones en las que el poder se entiende como el grado en el que un grupo es capaz de utilizar ciertos recursos políticos para su propio beneficio. Mi lista de recursos incluye 1) los cargos públicos, 2) ideas e información, 3) factores de producción (trabajo, tecnología y materias primas junto con el capital), 4) fuerza violenta, 5) grupos de activistas, y 6) autoridad moral. Cada recurso tiene un grupo prototipo asociado a él: el gobierno y la burocracia con los altos cargos públicos, los tecnócratas y los medios de comunicación con las ideas y la información, las empresas con los factores de producción, el ejército y la policía con la fuerza violenta, los partidos políticos con los activistas, y la autoridad moral con la Iglesia. Sin embargo, algunos grupos derivan su poder de más de un recurso: los partidos cuentan con activistas, ideas, autoridad moral (cuando son respetados) y los cargos públicos (cuando están en el gobierno); el gobierno cuenta con todos los recursos posibles en un momento o en otro. (COPPEDGE, 1994: 62-63).

Cualquier grupo que controle uno o más de estos recursos es potencialmente un actor estratégico siempre y cuando ese grupo demuestre una gran solidez. Para el autor antes citado los actores estratégicos de América Latina son el gobierno (los líderes políticos en la administración), el ejército, la burocracia y las empresas estatales, las cuales son conocidas colectivamente como el Estado; las asociaciones de empresarios, los sindicatos y confederaciones de trabajadores, las organizaciones de agricultores, la iglesia y otros grupos de interés, a los que se conoce colectivamente como la sociedad y los partidos políticos que intentan ser un mediador entre el Estado y la sociedad.

Añade que no es tan necesario que los actores estratégicos deban coincidir con los demás para que exista gobernabilidad, sino que lo que realmente importa es que acepten que no pueden mejorar su situación rechazando estos procedimientos a favor de otros o de ninguno (COPPEDGE, 1994: 63).

Luego de estas explicaciones COPPEDGE llega a una definición más precisa sobre gobernabilidad, señalando que "... la gobernabilidad es el grado en que las relaciones entre los actores estratégicos obedecen a fórmulas estables y mutuamente aceptadas" (1994: 63).

Para el autor, la lógica de la democracia es conflictiva para la gobernabilidad, ya que mientras la gobernabilidad requiere una representación efectiva de los grupos en proporción a su poder, la democracia requiere una representación efectiva de los grupos en relación al número de personas que los apoyan. De ahí que la "...gobernabilidad respete la lógica del poder, mientras que la democracia respeta la lógica de la igualdad política ..." (COPPEDGE, 1994: 64).

Para aclarar lo anteriormente señalado, el autor citado argumenta que en una democracia que encuentra su legitimidad a través de las elecciones, puede resultar verdaderamente peligroso que el Poder Ejecutivo trate de imponer obediencia en forma unilateral a todos los grupos.

Esto que desde una perspectiva democrática está total y plenamente justificado, puede ser fatal si se mira desde un punto de vista de la gobernabilidad. Es por ello que el autor señala que "... un mandato electoral no debería interpretarse como una licencia para imponer el deseo del pueblo sobre los intereses poderosos, sino 
como un mandato para representar al pueblo en las negociaciones con estos intereses poderosos..." (COPPEDGE, 1994: 63).

Analizando la teoría planteada por Coppedge, el autor Alcántara señala que de acuerdo con este autor existen seis (6) condiciones para la gobernabilidad. Las dos primeras se centran en el plano general ya que hacen alusión a "... la capacidad y el deseo de todos los grupos políticamente relevantes para comprometerse con algún tipo de arreglo institucional que sirviese para dirimir sus diferencias..."; mientras que las cuatro restantes se centran en los requisitos de la gobernabilidad democrática.

Estos requisitos están referidos a:

...1) aceptación de compromisos institucionales democráticos que confieren gran peso a los grupos de masa políticamente relevantes (básicamente los partidos políticos) por parte de aquellos otros de carácter más elitista (Iglesia, empresarios, militares, etcétera); 2) la aceptación de arreglos que permitan la representación efectiva de estos últimos grupos por parte de la clase política elegida; 3) la efectiva representación de los ciudadanos por esta clase política elegida; 4) la creación y el mantenimiento de mayorías que funcionen basadas en criterios partidistas como consecuencia de decisiones tomadas por la clase política" (ALCÁNTARA, 1995: 31).

\section{LA INFLUENCIA DEL PODER PRESIDENCIAL, EL PRESIDENTE ES: ¿LÍDER O EMPLEADO?}

El autor NEUSTADT, (1993: 32) en su obra "El Poder Presidencial", o la Influencia del Presidente como señala en sus líneas, explica que la forma de evaluar a un Presidente es medida en términos de debilidad o fortaleza, esa medida la llama liderazgo; indica el autor que hay dos (2) formas de estudiar el "poder presidencial", a saber: Una es centrarse en las tácticas (...) de influir en ciertos hombres en situaciones dadas: como el obtener que el Congreso apruebe una ley, como arreglar huelgas, como silenciar rencillas del gabinete, o como detener casos ( ... ). La otra manera es retraerse de las tácticas en los casos dados y ocuparse de la influencia en términos más estratégicos: el autor se pregunta ¿Cuál es su naturaleza y cuáles son sus fuentes?, ¿Qué puede este hombre hacer para mejorar la posibilidad de tener influencia cuando lo desee? Estratégicamente, la cuestión no es cómo maneja al Congreso en una situación dada, sino qué hace para realzar su posibilidad de manejo en cualquier instancia, mirando al mañana desde el presente.

Un aspecto que señala el autor citado, es que para investigar la estrategia de la influencia presidencial hay que decidir primero a quien investigar, generalmente se buscan los presidentes más recientes; indica además que las apariencias cambian pero las etiquetas permanecen iguales y también los dilemas. Con el término emergencia describe las condiciones; los dilemas de la política se asemejan al pasado en un aspecto: su dificultad y complejidad para gobernarlos. Se imponen enormes tensiones "... En nuestro tiempo la innovación nos mantiene perfectamente confusos; tan pronto comenzamos a comprender algo, otra cosa nueva se le añade y andamos a 
tientas otra vez ..." (NEUSTADT, 1993: 33).

Los dilemas políticos rara vez producen lo que el país siente como una crisis; los métodos políticos han sido los habituales, pero solo según la norma de las crisis del pasado. Las crisis presentes son: el debilitamiento de los partidos políticos, el énfasis en la personalidad, la estrecha cercanía de los acontecimientos mundiales, la volubilidad del público y, sobre todo, el corte de boletas.

Se comparan entonces a presidentes de mitad de siglo, Franklin Roosevelt, quien tenía una situación bastante comparable hacia la mitad de su presidencia y Abraham Lincoln "... que está mucho más cerca de nosotros por las condiciones que lo rodeaban ...", que por la época. (NEUSTADT, 1993: 34). Y Woodrow Wilson se enfrentó y fue derrotado por condiciones semejantes a las actuales. En cambio Truman y Eisenhower fueron los primeros en dar forma a la influencia presidencial.

NEUSTADT, (1993), considera que el liderazgo puede consistir en una serie de iniciativas en lo económico diplomático, legislativo o en la comunicación de masas. Los presidentes de mitad de siglo han dado todas las muestras de lo que tradicionalmente se asocia a la fortaleza del cargo. Theodore Roosevelt asumió una vez el rol de administrador en la situación de emergencia creada por la gran huelga del carbón en 1902, (la Ley Laboral del Ferrocarril y la Ley Taft-Hartley han convertido esas intervenciones obligatorias para los presidentes), en su siguiente presidencia hizo valer una vez su responsabilidad personal para amojonar y guiar la economía norteamericana (la Ley del Empleo obliga a sus sucesores a esa tarea); Wilson y Franklin Roosevelt se hicieron grandes oradores, actores protagónicos en la escena mundial en un momento culminante de guerra (Ley de Seguridad Nacional). Estos presidentes se movieron a tientas con la necesidad creada por la nueva emergencia, de integrar intereses militares y políticos. Franklin Roosevelt y Truman se hicieron responsables del desarrollo y uso primero de armas atómicas (Ley de Energía Atómica).

Formalmente todos los presidentes son líderes en la actualidad, lo que garantiza que servirán como funcionarios, esto no significa que el resto del gobierno está a los pies del hombre, sino que otros hombres han encontrado prácticamente imposible hacer lo que les corresponde sin la seguridad de las iniciativas del presidente. El servicio a ellos mismos, no el poder para el presidente, es lo que os lleva a aceptar este liderazgo formal. Entonces un presidente en estos días es un valioso empleado. Por ello, los funcionarios ejecutivos necesitan decisiones, protección política y un árbitro en las peleas.

Un presidente moderno está obligado, según NEUSTADT (1993: 36-37), a enfrentar demandas de ayuda y servicios de más o menos cinco (5) procedencias distinguibles: (1) de los funcionarios ejecutivos, (2) del Congreso, (3) de sus partidarios, (4) de los ciudadanos en general y (5) del exterior. Se los distingue más bien por los diferentes reclamos sobre el presidente, lo que quieren son iniciativas, por cinco razones diferentes. Y finaliza el autor, pero, precisamente por ser "único", recaen sobre él solo. Las mismas condiciones que promueven su 
liderazgo de forma son las que impiden una garantía del liderazgo de hecho (cursivas de la cita). Por eso es que sus servicios están en demanda; las obligaciones de todos los hombres son diferentes a las de él, todos tienen diferentes tareas y no pueden ver las obligaciones de aquel como propias y ninguna es parecida a la que aquel tiene, cuando se percatan de sus tareas pueden encontrar que lo correcto es seguir o no seguir al presidente.

\section{CONSIDERACIONES FINALES}

Luego de la revisión bibliográfica realizada a través de diferentes autores que tratan el tema de la gobernabilidad puede concluirse que el concepto de gobernabilidad, se basa inicialmente en un análisis de la estabilidad política y a las relaciones entre actores e instituciones políticas y esta además referido al equilibrio dinámico entre demandas sociales y respuestas gubernamentales/estatales.

Además la gobernabilidad se relaciona con la legitimidad y la eficacia, transformándose en las variables independientes que se deben considerar en todo estudio relacionado con la gobernabilidad.

Los autores Pasquino, Camps y Boeninger tratan la gobernabilidad como un concepto en el que están inmersos actores sociales y públicos, donde debe existir interacción entre ellos en función del rol que cada uno de los actores debe jugar en un sistema democrático

Para aclarar lo anteriormente señalado, el autor citado argumenta que en una democracia que encuentra su legitimidad a través de las elecciones, puede resultar verdaderamente peligroso que el Poder Ejecutivo trate de imponer obediencia en forma unilateral a todos los grupos.

Actualmente a los gobiernos ya no les basta con tener legitimidad de origen, sino que deben ganársela día a día siendo eficaces en las medidas que adoptan, sobre todo en un momento en que la discusión se centra en definir cuál es el rol que le compete al Estado en el nuevo escenario internacional, situación que se ha agudizado a partir de la década de los ochenta como resultado de la implantación del modelo neoliberal. Por ello es muy importante el papel que juega el Presidente como líder, ya que a medida que surjan las crisis se medirá la capacidad de su liderazgo así como el rol de los actores y las medidas aplicadas.

\section{GOVERNANCE OR ABILITY TO GOVERN. THE ROLE OF POLITICAL LEADERSHIP}

\section{Abstract}

The present work has as objective to analyze the concept of governability, seen from the perspective of theoreticians like Alcántara, Arbós and Giner, Huntington, Bell, Nohlen and Neudstadt, among others. Governability has been defined as the capacity to govern, and this capacity can be limited by crises than by democratic systems that are given by internal factors (political parties, society) and external factors (global environment), where efficiency and legitimacy play an important role within the system. As for the evaluation of 
the leader through leadership, the role played by the President as leader is very important, since as the crises arise the capacity of his leadership will be measured as well as the role of the actors, the measures applied and the emphasis on its followers.

Keywords: governance, leadership, management, democracy and political science.

\section{BIBLIOGRAFÍA}

ALCÁNTARA, Manuel. (1994). “De la gobernabilidad”. Revista América Latina Hoy No 8, Instituto de Estudios de Iberoamérica y Portugal, Universidad de Salamanca, Salamanca.

ALCÁNTARA, Manuel. (2004). Gobernabilidad, crisis y cambio. Elementos para el estudio de la gobernabilidad de los sistemas políticos en épocas de crisis y cambio. Fondo de Cultura Económica. México.

ARBÓS, Xavier y Giner, Salvador. (1996). La gobernabilidad. Ciudadanía y democracia en la encrucijada mundial Editorial Siglo XXI, Madrid.

BOENINGER, Edgardo. (1994). La Gobernabilidad: un concepto multidimensional. En Luciano Tomassini (comp.), ¿Qué espera la sociedad del gobierno?, Centro de Análisis de Políticas Públicas, Asociación Chilena de Ciencia Política, Santiago de Chile.

(1997) Democracia en Chile. Lecciones para la gobernabilidad. Editorial Andrés Bello, Santiago de Chile.

BUCHANAN, James M. y Wagner, Richard E. (1983) Déficit del sector público y democracia. El legado político de lord Keynes. Editorial Rialp, S.A., Madrid.

CAMOU, Antonio. (2001). (Estudio preliminar y compilación) (2001). Los desafíos de la Gobernabilidad. México: Flacso/IISUNAM/Plaza y Valdés.

CAMPS, Victoria. (1997) "Ética del Buen Gobierno". En Salvador Giner y Sebastián Sarasa (edits.), Buen gobierno y política social. Editorial Ariel, S.A., Barcelona.

COPPEDGE, Michael. (1994) “Instituciones y gobernabilidad democrática en América Latina”, Revista Síntesis № 22. Sociedad Editorial Síntesis, S.A., Madrid, 1994.

FLISFISCH, Ángel (1989). “Gobernabilidad y consolidación democrática: sugerencias para la discusión”, Revista Mexicana de Sociología, LI. 3. En Manuel Alcántara, Revista América Latina Hoy No 8, Instituto de Estudios de Iberoamérica y Portugal, Universidad de Salamanca, Salamanca, 1994.

FRANCO, Rolando. (1994) "Estado, consolidación democrática y gobernabilidad en América Latina", Revista América Latina Hoy No 8, Instituto de Estudios de Iberoamérica y Portugal, Universidad de Salamanca, Salamanca, 1994.

HAYEK, Friedrich A. (1995). Camino de servidumbre. Editorial Alianza S.A., Madrid. 
HEREDIA V., Raimundo. (2002). Gobernabilidad: una aproximación teórica. VII Congreso Internacional del CLAD sobre la Reforma del Estado y de la Administración Pública, Lisboa, Portugal, 8-11 Oct. 2002, páginas 117.

HUNTINGTON, Samuel (1968). Political Order in Changing Societies, New Haven, Conn; Yale University Press. En Michael Coppedge, "Instituciones y gobernabilidad democrática en América Latina”, Revista Síntesis No 22, Sociedad Editorial Síntesis, S.A., Madrid, 1994. Para este trabajo, no obstante, se utilizó la cuarta reimpresión en castellano publicada por Editorial Piados en 1997.

LINZ, Juan. (1996). La quiebra de las democracias. Editorial Alianza Universidad.

LIPSET, Seymour Martin. (1992) Algunos requisitos sociales de la democracia: desarrollo económico y legitimidad política. En Diez Textos Básicos de Ciencia Política. Editorial Ariel, Barcelona.

(1996). "Repensando los requisitos sociales de la democracia", Revista Ágora nº 5, Universidad de Buenos Aires, Buenos Aires.

MORLINO, Leonardo. (1985). Cómo cambian los regímenes políticos. Centro de Estudios Constitucionales, Madrid.

NEUSTADT, Richard E., (1993). El poder presidencial y los presidentes modernos, Bs. As., Grupo Editor Latinoamericano

NOHLEN, Dieter. (1994) “Sistemas electorales y gobernabilidad”, Working Paper 63. Institut de Ciénces Politiques y Social. Barcelona. En Manuel Alcántara, Revista América Latina Hoy No 8, Instituto de Estudios de Iberoamérica y Portugal, Universidad de Salamanca, Salamanca.

O’CONNOR, James. (1981) La crisis fiscal del Estado. Editorial Península, Barcelona, 1981.

PASQUINO, Gianfranco. (1997) “Gobernabilidad y calidad de la democracia”. En Salvador Giner y Sebastián Sarasa (edits.), Buen gobierno y política social. Editorial Ariel, S.A., Barcelona.

TOMASSINI, Luciano. (1996). "La gobernabilidad en América Latina”. Revista Síntesis No 25, Sociedad Editorial Síntesis, Madrid.

TORRES SILVA, Edelberto. (1994). "La gobernabilidad centroamericana en la década de los '90. Consideraciones sobre las posibilidades democráticas en la postguerra”. Revista América Latina Hoy No 8, Instituto de Estudios de Iberoamérica y Portugal, Universidad de Salamanca, Salamanca.

WEBER, Max. (1994). Economía y sociedad. Editorial Fondo de Cultura Económica, México.

ZUMBADO, Fernando. (1994). La gobernabilidad y la modernización del Estado democrático. En Luciano Tomassini (comp.), ¿Qué espera la sociedad del gobierno?, Centro de Análisis de Políticas Públicas, Asociación Chilena de Ciencia Política, Santiago de Chile.

Trabalho enviado em 18 de setembro de 2017.

Aceito em 01 de janeiro de 2018. 